\title{
Bioinspired single-chain polymer nanoparticles
}

\author{
José A. Pomposo ${ }^{a, b, c^{*}}$ \\ "Correspondence to: José A. Pomposo, Centro de Física de Materiales (CSIC, UPV/EHU)-Materials Physics Center, Paseo Manuel de Lardizabal 5, 20018 \\ San Sebastián, Spain E-mail: Josetxo.pomposo@ehu.es
}

${ }^{a}$ Centro de Física de Materiales (CSIC, UPV/EHU)-Materials Physics Center, Paseo Manuel de Lardizabal 5, 20018 San Sebastián, Spain

${ }^{b}$ Departamento de Física de Materiales, Universidad del País Vasco (UPV/EHU), Apartado 1072, 20800 San Sebastián, Spain

'IKERBASQUE - Basque Foundation for Science, Alameda Urquijo 36, 48011 Bilbao, Spain

\begin{abstract}
Polymer chains can be folded / collapsed to individual, single-chain polymer nanoparticles (SCNPs) by means of intrachain crosslinking techniques. In some way, SCNP formation is reminiscent of protein folding although current synthetic methods lack the perfection of protein folding to functional enzymes. However, in recent years the structure-function paradigm (i.e., amino-acid sequence $\rightarrow$ 3D structure $\rightarrow$ function) has been revisited by taking into account that many non-structured segments of proteins, and even totally disordered proteins, play important roles in protein function. In this perspective article, we highlight the significant added value that is endowed to SCNPs by taking inspiration from the functions of both ordered and disordered proteins.
\end{abstract}

Keywords: single-chain polymer nanoparticles; folding / collapse; ordered / disordered proteins; biomimetic nano-objects

\section{INTRODUCTION}

Control over the composition, size, shape and functionality of hard, synthetic nano-objects has grown exponentially in recent years. The huge interest in hard nanoparticles (e.g., metallic, metal oxides) has been a direct consequence of several new, nano-scale effects found in hard nanosized materials when compared to their bulk counterparts. ${ }^{1} \mathrm{~A}$ classical example is that concerning spherical gold nanoparticles, showing a specific UV-visible Plasmon resonance band as a consequence of electron surface confinement, which can be easily tuned by changing nanoparticle size and shape. The huge surface-tovolume ratio in hard nano-objects also contributes to the enhanced reactivity and particular properties often displayed by nanomaterials, when compared to those of classical micronsized particles (Fig. 1(a)).

Concerning artificial soft nano-objects, significant advances have been performed in the last decades in the control of macromolecular architectures (composition, size, shape, dispersity) thanks, mainly, to the development of efficient controlled radical polymerization (CRP) techniques like nitroxide-mediated radical polymerization (NMP), ${ }^{2}$ atomtransfer radical polymerization (ATRP) ${ }^{3}$ and radical addition fragmentation chain-transfer (RAFT) polymerization. ${ }^{4}$ In fact, several complex architectures such as those in stars polymers, comb-like copolymers and hyperbranched macromolecules have been generated using CRP techniques; ${ }^{5-8}$ the resulting materials possessing properties very different from that of their linear counterparts of equivalent molecular weight (Fig. 1(b)). A particular class of artificial soft nano-objects is single-chain polymer nanoparticles (SCPNs), which are individual intrachain cross-linked polymer chains that have attracted significant attention in the polymer field and the nanoscience arena. ${ }^{9-11}$ One of the main characteristics of SCNPs when compared to conventional latex particles is their ultra-small size $(<15 \mathrm{~nm})$ and consequently their inherent large surface-to-volume ratio as well as absence of any emulsifier during their synthesis at high dilution. Different techniques have been introduced in the past years for intrachain folding/collapse of individual polymer chains to SCNPs, such as intrachain homocoupling, intrachain heterocoupling and cross-linking induced collapse. ${ }^{9}$ Additionally, covalent bonding and non-covalent/dynamiccovalent bonding interactions have been used during the collapse of linear polymeric precursors to permanent and reversible/stimuli-responsive single-chain nanoparticles, respectively. ${ }^{10,11}$ First generations of SCNPs were developed for use as sacrificial porogens in microelectronics ${ }^{12}$ and as rheology improvers in polymer blends. ${ }^{13-15}$ In this perspective article, we highlight the significant added value that can be endowed to SCNPs by taking inspiration from the functions of both ordered and disordered proteins (Fig. 1(c)). 


\section{(a) HARD, SYNTHETIC NANO-OBJECTS}

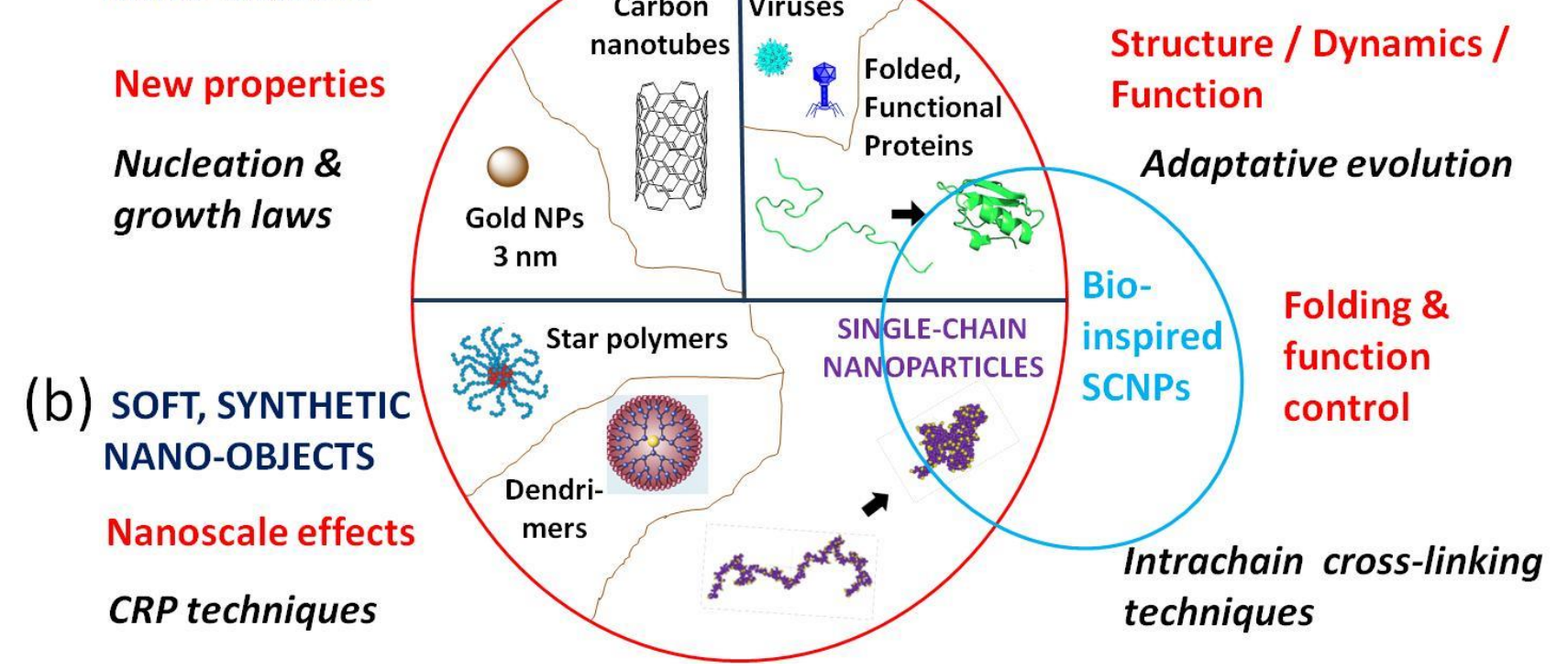

Figure 1. (a) Interest in hard, synthetic nano-objects like gold nanoparticles or carbon nanotubes was driven by the interesting nano-scale properties showed by these nanosized particles when compared to the corresponding bulk materials. ${ }^{1}$ (b) Controlled radical polymerization (CRP) techniques ${ }^{2-4}$ paved the way to the synthesis of soft, synthetic nano-objects of complex architecture like stars polymers, comb-like copolymers, hyperbranched polymers ${ }^{5-8}$ or single-chain polymer nanoparticles (SCNPs). ${ }^{9-11}$ The folding/collapse process during SCNP formation is reminiscent of protein folding to the native functional state, although current SCNPs mimic the globular structure of biomacromolecules only in a very rough, primitive manner. ${ }^{9-11}$ (c) Biomimetic SCNPs can be constructed by taking inspiration from the functions of soft, natural nano-objects and, in particular, from both folded proteins ${ }^{16-}$ 20 as well as intrinsically disordered proteins (IDPs). ${ }^{21-25}$

\section{TAKING INSPIRATION FROM PROTEINS}

The overall shape of proteins is determined, mainly, by the precise sequence of amino-acids, the interactions between amino-acids in solution as well as the interactions of protein residues with water. As a result, in Nature we can find from perfectly ordered (or folded) proteins ${ }^{16-20}$ to intrinsically disordered (unfolded or coil-like) proteins. ${ }^{21-25}$ Sometimes, disordered flexible linkers are found to bind folded domains. In recent years, the structure-function paradigm (i.e., amino-acid sequence $\rightarrow 3 D$ structure $\rightarrow$ function) has been revisited by taking into account that many non-structured segments of proteins, and even totally disordered proteins, play important roles in protein function. ${ }^{21-24}$ Many globular proteins are enzymes which catalyze biochemical reactions via active sites placed in a hydrophobic core, whereas the function of intrinsically disordered proteins is often to bind ligands, such as ions, small organic molecules, other proteins and nucleic acids (RNA, DNA). ${ }^{25}$ Examples of proteins coming from fungi, bacteria and human body which are involved in specific biological processes such as oxidation (Lacase enzyme), polymerization (Taq polymerase) and transport (Vitamin-D binding protein) are illustrated in Fig. 2. As we will show in next section, all of them have been recently valuable sources of inspiration for the construction of biomimetic SCNPs.

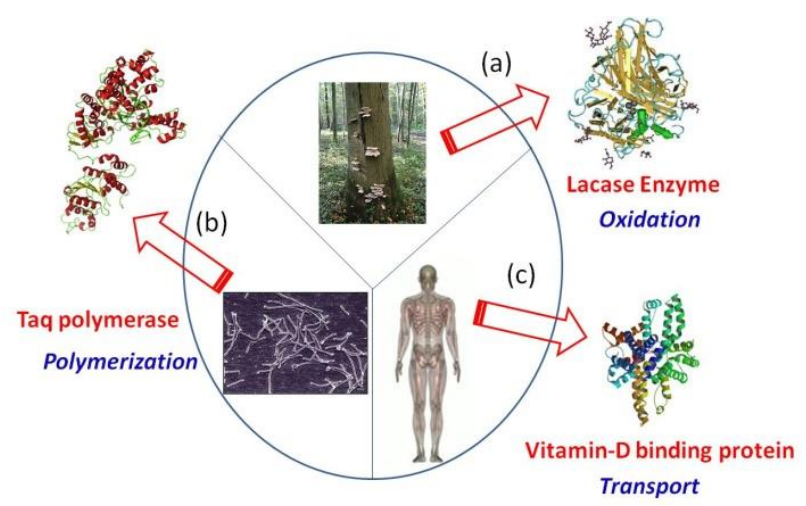

Figure 2. Examples of several proteins with specific biological functions which can be taken as source of inspiration for the construction of functional biomimetic SCNPs: (a) Lacase enzyme from Pleurotus ostreatus. (b) Taq polymerase from Thermus aquaticus. (c) Vitamin-D binding protein (DBP), also known as GC-globulin, from human serum.

\section{BIOMIMETIC SINGLE-CHAIN POLYMER NANOPARTICLES}

Since most SCNP precursors are random copolymers lacking a precise monomer sequence, the shape of SCNPs in solution, when compared to that of proteins, depends mainly on the efficiency of the intrachain cross-linking technique employed as well as the subtle balance of SCNP-solvent interactions. Hence, for SCNPs in solution one can expect a range of structures from 


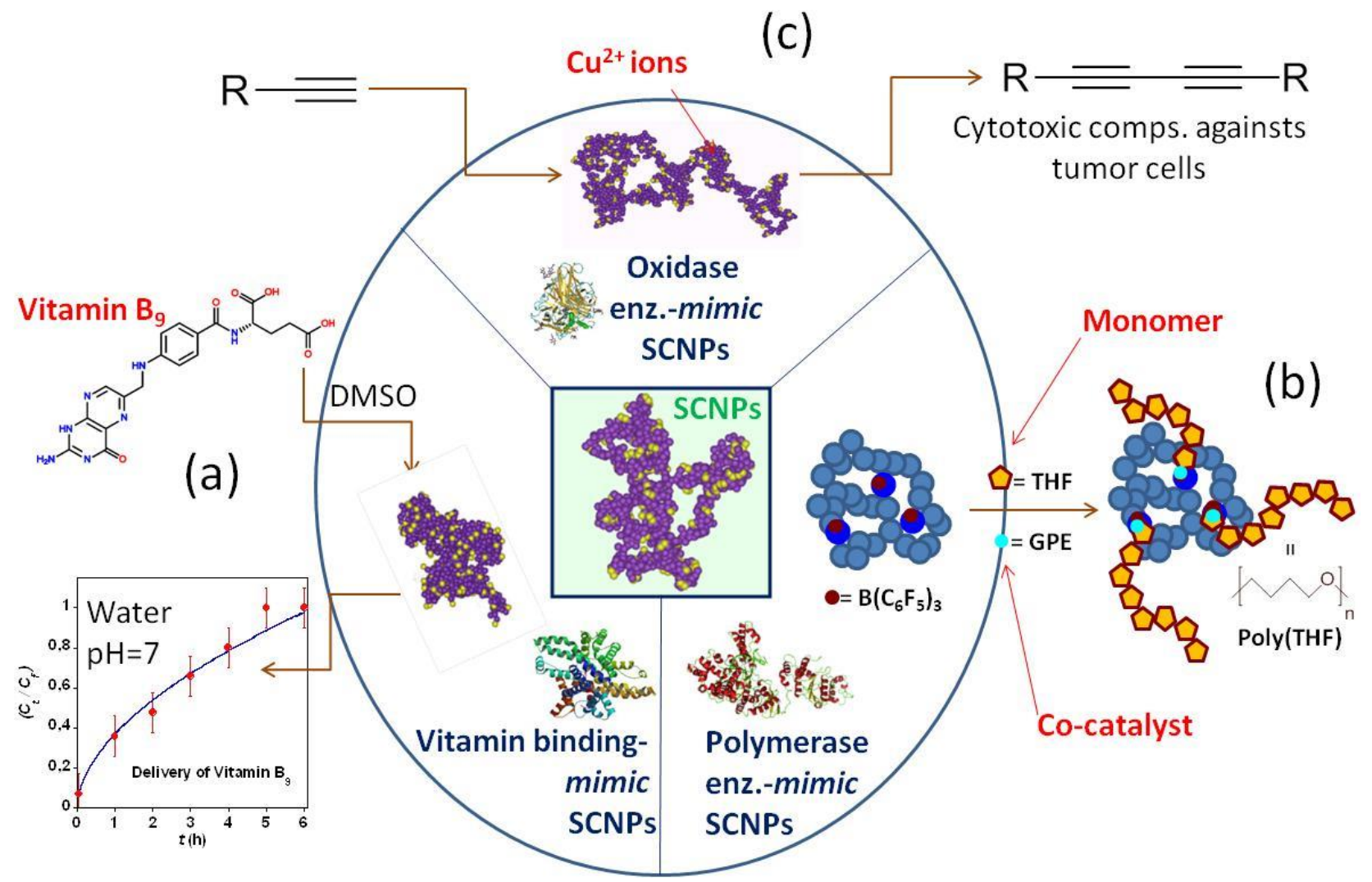

Figure 3. (a) Vitamin binding-mimic SCNPs synthesized via Michael addition-mediated multidirectional self-assembly ${ }^{28}$ are novel transient-binding disordered nanocarriers from which controlled delivery of both dermal protective (vitamin $\mathrm{B}_{9}$ ) and anticancer (hinokitiol) cargos can be carried out. ${ }^{29}$ (b) Dual reductase- / polymerase-enzyme-mimic SCNPs have been synthesized through a new approach ${ }^{30}$ which results in non-globular catalytic nano-objects allowing highly-efficient reductions to be performed (turnover frequency, TOF $>5800 \mathrm{~h}^{-1}$ ) as well as the synthesis of high-molecular-weight poly(tetrahydrofuran), poly(THF), in the presence of glycidyl phenyl ether (GPE) in an enzyme-like fashion. (c) Oxidase enzyme-mimic SCNPs synthesized via $\mathrm{Cu}^{2+}$ intrachain cross-linking show a disordered structure in solution and allow chemoselective alkyne homocoupling reactions to be performed, the resulting compounds being useful as cytotoxic agents against tumor cells.

disordered, crumpled conformations to pseudo-globular morphologies. The important point to stress here is that by analogy to proteins, even disordered non-globular SCNPs can show interesting functions related to those of natural biomolecules.

By taking inspiration from globular enzymes, SCNPs having a hydrophobic compartment containing the catalytic species have been recently reported by Meijer and co-workers. ${ }^{26,27}$ Hence, water-soluble single-chain poly(dodecyl methacrylate)based nanoparticles having a catalytically active Ru-containing hydrophobic cavity were synthesized from amphipilic $A B A$ terpolymers $(A=\operatorname{poly}($ ethylene glycol) methyl ether methacrylate block, $\mathrm{B}=$ random block of dodecyl methacrylate and BTA-bearing methacrylate repeat units; BTA = chiral benzene-1,3,5-tricarboxamide). By using these biomimetic nanoparticles at a [substrate] / [Ru] ratio of $200 / 1$, the quantitative reduction of cyclohexanone to cyclohexanol in water was carried out in $18 \mathrm{~h}$ with turnover frequency (TOF) values $\left(\right.$ TOF $=10-20 \mathrm{~h}^{-1}$ ) similar to those reported for conventional water-soluble Ru-complexes $\left(\mathrm{TOF}=1-40 \mathrm{~h}^{-1}\right) .^{26}$ In a subsequent use of this "hydrophobic cavity" approach, ${ }^{27} \mathrm{~L}$ proline (L-Pro)-funcionalizated random terpolymers synthesized from L-Pro-bearing methacrylate (5 mol\%), BTA-bearing methacrylate (10 mol\%) and oligo(ethylene) glycol methacrylate monomers were transformed into single-chain nanoparticles via helical stacking of the chiral BTA units, and used as efficient catalysts for the aldol reaction in water between $p$-nitrobenzaldehyde and cyclohexanone, showing high conversion (99\% after 120 h), high diastereoselectivity (92\%) but moderate enantioselectivity (73\%). Bioinspired construction of hydrophobic cavity-containing SCNPs is then a new avenue for enzyme-like organic chemistry in water from which many new contributions are expected to appear in a near future (e.g., multi-step cascade reactions in water).

On the other hand, SCNPs showing form factors in solution similar to those of intrinsically disordered proteins (IDPs) have been recently synthesized by Pomposo and co-workers ${ }^{28-30}$ and investigated for different biomimetic applications. In a first 
example of bioinspired nanocarriers, single-chain nanoparticles were synthesized via Michael addition-mediated multidirectional self-assembly from precursors containing $\beta$ ketoester functional groups. Small angle neutron scattering (SANS) measurements combined with molecular dynamics (MD) simulations undoubtedly showed that the form factor of these SCNPs in solution resembles that of IDPs instead of that expected for globular enzymes. ${ }^{28}$ Even without the precise sequence of proteins, the mimicking of IDP morphology in good solvent was a consequence of the intrachain self-assembly process leading to the formation of local globules along the individual polymer chains. The resulting SCNPs were used as novel transient-binding disordered nanocarriers (see Fig. 3(a)) from which controlled delivery of both dermal protective (vitamin $\mathrm{B}_{9}$ ) and anticancer (hinokitiol) cargos in water was carried out. ${ }^{29}$ It is worth of mention that these "Michael" nanocarriers adopted a collapsed, globular morphology in the dry state, as observed by transmission electron microscopy (TEM). This extended-to-compact morphology transition taking place upon solvent removal could be of paramount importance, among other applications, for the construction of efficient biosensors based on immobilized protein-mimic nano-objects.

In a second example, dual reductase-/polymerase- enzymemimic SCNPs were synthesized through a new approach which results in catalytic nano-objects allowing highly-efficient reductions to be performed (TOF > $5800 \mathrm{~h}^{-1}$ ) as well as the synthesis of high-molecular-weight poly(tetrahydrofuran), poly(THF) in the presence of glycidyl phenyl ether (GPE) in an enzyme-like fashion (Fig. 3(b)). ${ }^{30}$ This new pathway to SCNPs endowed with enzyme-mimetic activity was based on "concurrent" catalyst-assisted intramolecular cross-linking of linear precursors and concomitant binding of the catalyst to multiple SCNP intrachain cross-linked sites (i.e, "concurrent" approach). Hence, instead of possessing an isolated compact hydrophobic compartment, ${ }^{26,27}$ the resulting SCNPs showed multiple, compartmentalized local catalytic sites as well as a relatively open/extended morphology in solution, as determined by SANS experiments.

As a last example of bioinspired SCNPs, oxidase enzymemimic SCNPs have been recently synthesized in our laboratory via $\mathrm{Cu}^{2+}$-mediated intrachain cross-linking showing a disordered crumpled structure in solution as illustrated schematically in Fig. 3(c). In spite of the lack of globular order, as found e.g. in Lacase enzyme, these SCNPs synthesized using the novel "concurrent" approach allow performing chemoselective alkyne homocoupling reactions, the resulting compounds being useful as cytotoxic agents against tumor cells.

\section{SUMMARY}

Folding of individual polymer chains to collapsed SCNPs is reminiscent of protein folding and, currently, it can be performed by means of different intrachain cross-linking techniques. In this perspective article we have shown how the potential applications of SCNPs broaden significantly by taking inspiration from the functions of both ordered and disordered proteins. On one hand, by taking inspiration from globular enzymes, SCNPs having a hydrophobic compartment containing the catalytic species have been reported, paving the way to enzyme-like organic chemistry in water. On the other hand, by taking inspiration from IDPs, transient-binding disordered SCNPs have been recently synthesized, from which controlled delivery of both dermal protective and anticancer cargos has been carried out. Moreover, the recent introduction of a novel "concurrent" approach has opened the door to SCNPs mimicking the structure of IDPs having reductase-/polymeraseand oxidase-like properties, respectively. Due to the added value that is endowed to SCNPs by taking inspiration from the functions of both ordered and disordered proteins, we anticipate further developments coming soon in the still emerging field of bioinspired SCNPs.

\section{ACKNOWLEDGEMENTS}

Financial support from the projects MAT2012-31088 (MINECO) and IT-654-13 (GV) is acknowledged.

\section{REFERENCES}

1 Grzelczak M, Vermant J, Furst EM and Liz-Marzan LM, ACS Nano 4: 3591-3605 (2010).

2 Hawker CJ, Bosman AW and Harth E, Chem. Rev. 101: 3661 3688 (2001).

3 Matyjaszewski K and Xia JH, Chem. Rev. 101: 2921-2990 (2001).

4 Moad G, Rizzardo E and Thang SH, Aust. J. Chem. 58: 379-410 (2005).

5 Taton D, Gnanou Y, Matmour R, Angot S, Hou S, Francis R, Lepoittevin B, Moinard D and Babin J, Polym. Int. 55: 1138-1145 (2006).

6 Hadjichristidis N, latrou H, Pitsikalis M and Mays J, Prog. Polym. Sci. 31: 1068-1132 (2006).

7 Matyjaszewski K, Nature Chem. 1: 276-288 (2009).

8 Gregory A and Stenzel MH, Prog. Polym. Sci. 37: 38-105 (2012).

9 Sanchez-Sanchez A, Perez-Baena I and Pomposo JA, Molecules 18: 3339-3355 (2013).

10 Altintas $\mathrm{O}$ and Barner-Kowollik C, Macromol. Rapid Commun. 33: 958-971 (2012).

11 Sanchez-Sanchez A and Pomposo JA, Part. Part. Syst. Charact. In press, DOI: 10.1002/ppsc.201300245.

12 Mecerreyes D, Lee V, Hawker CJ, Hedrick JL, Wursch A, Volksen W, Magbitang T, Huang E and Miller RD, Adv. Mater. 13: 204-208 (2001).

13 Mackay ME, Dao TT, Tuteja A, Ho DL, Van Horn B, Kim HC and Hawker CJ, Nature Mater. 2: 762-766 (2003).

14 Tuteja A, Mackay ME, Hawker CJ and Van Horn B, Macromolecules 38: 8000-8011 (2005).

15 Mackay ME, Tuteja A, Duxbury PM, Hawker CJ, Van Horn B, Guan ZB, Chen GH and Krishnan RS, Science 311: 17401743 (2006).

16 Dill KA and MacCallum JL, Science 338: 1042-1046 (2012). 
17 Hartl FU and Hayer-Hartl M, Science 295: 1852-1858 (2002).

18 Hardesty B and Kramer G, Prog. Nucleic Acid Res. Mol. Biol. 66: 41-66 (2001).

19 Baker D, Nature 405: 39-42 (2000).

20 Baldwin RL, Nature 369: 183-184 (1994).

21 Uversky VN, BBA-Proteins. Proteom. 1834: 932-951 (2013).

22 Tompa P, Trends Biochem. Sci. 27: 527-533 (2002).

23 Dunker AK, Brown CJ, Lawson JD, lakoucheva LM and Obradovic Z, Biochemistry-UK 41: 6573-6582 (2002).

24 Wright PE and Dyson HJ, J. Mol. Biol. 293: 321-331 (1999).

25 Tompa P and Han KH, Phys. Today 65: 64-65 (2012).

26 Terashima T, Mes T, De Greef TFA, Gillissen MAJ, Besenius P, Palmans ARA and Meijer EW, J. Am. Chem. Soc. 133: 4742-4745 (2011).

27 Huerta E, Stals PJM, Meijer EW and Palmans ARA, Angew. Chem. Int. Ed. 52: 2906-2910 (2013).

28 Sanchez-Sanchez A, Akbari S, Etxeberria A, Arbe A, Gasser U, Moreno AJ, Colmenero J and Pomposo JA, ACS Macro Lett. 2: 491-495 (2013).

29 Sanchez-Sanchez A, Akbari S, Moreno AJ, Verso FL, Arbe A, Colmenero J and Pomposo JA, Macromol. Rapid Commun. 34: 1681-1686 (2013).

30 Perez-Baena I, Barroso-Bujans F, Gasser U, Arbe A, Moreno AJ, Colmenero J and Pomposo JA, ACS Macro Lett. 2: 775779 (2013). 